\title{
Imaging of the Shoulder Bankart Lesion and its Variants
}

\author{
Leow KS ${ }^{1}$, Low SF ${ }^{2}$, PehWCG \\ ${ }^{1}$ Department of Diagnostic Radiology, Khoo Teck Puat Hospital, Yishun Central, Singapore, \\ Republic of Singapore \\ ${ }^{2}$ Department of Radiology, Kuala Lumpur Sports Medicine Centre, Jalan Dungun, Bukit \\ Damansara, Kuala Lumpur, Malaysia
}

Received: March 08, 2019

Accepted: April 15, 2019

Published: June 30, 2019

Cite this paper:

Leow KS, Low SF, PehWCG. Imaging of the Shoulder Bankart Lesion and its Variants. Nepalese Journal of Radiology 2019;9(13):33-39. https://doi.org/10.3126/njr.v9i1.24816

\begin{abstract}
The glenoid labrum is an important soft tissue structure that provides stability to the shoulder joint. When the labrum is injured, affected patients may present with chronic shoulder instability and future recurrent dislocation. The Bankart lesion is the most common labral injury, and is often accompanied by a Hill-Sachs lesion of the humerus. Various imaging techniques are available for detection of the Bankart lesion and its variants, such as anterior labroligamentous periosteal sleeve avulsion and Perthes lesion. Direct magnetic resonance (MR) arthrography is currently the imaging modality of choice for evaluation of the various types of labral tears. As normal anatomical variants of glenoid labrum are not uncommonly encountered, familiarity with appearances of this potential pitfall helps avoid misdiagnosis.
\end{abstract}

Key words: Anterior Labroligamentous Periosteal Sleeve Avulsion, Glenohumeral Joint Dislocation, Hill-Sachs Lesion, MR Arthrography, Perthes Lesion, Shoulder Dislocation

\section{INTRODUCTION}

The shoulder joint is one of the more commonly injured joints in the body, with its inherently large range of mobility predisposing it to risk of dislocation and development of chronic instability. Anterior glenohumeral dislocation is the most common form of acute traumatic instability, accounting for more than $95 \%$ of glenohumeral joint dislocations. Imaging has an important role in the evaluation of shoulder injuries and associated internal derangements, with magnetic resonance (MR) imaging being the modality of choice.

\section{ANATOMICAL CONSIDERATIONS}

Correspondence to: Prof. Wilfred CG Peh

Clinical Professor and Head

Department of Diagnostic Radiology

Khoo Teck Puat Hospital, 90 Yishun Central

Singapore 768828, Republic of Singapore

Email: Wilfred.peh@gmail.com

The glenohumeral joint is a ball and socket joint which formed by the glenoid fossa of the scapula together with the humeral head. The glenoid fossa is pear-shaped in configuration and is relatively shallow, which allows a great

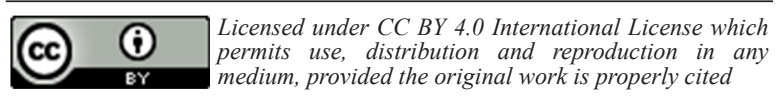


range of shoulder movement. The stability of the glenohumeral joint depends on the glenoid labrum, glenohumeral ligaments and rotator cuff musculotendinous structures. The glenoid labrum consists of a fibrocartilaginous rim which attaches circumferentially around the glenoid fossa (Figure 1a). The glenoid labrum is an important structure as it enables deepening of the glenoid cavity by approximately $50 \%{ }^{1}$ The labrum also serves as the attachment site for glenohumeral ligaments and long head of biceps tendon (LHBT). There are three glenohumeral ligaments: the superior, middle and inferior glenohumeral ligaments, which are formed by thickenings of the joint capsule. The anterior band of the inferior glenohumeral ligament (IGHL) attaches to the anteroinferior labrum and is recognized as the main structure

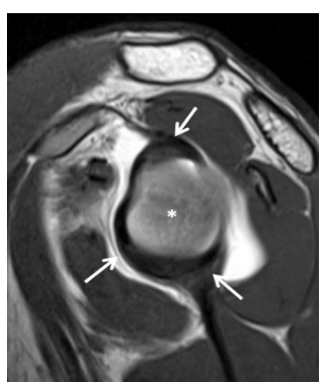

1a

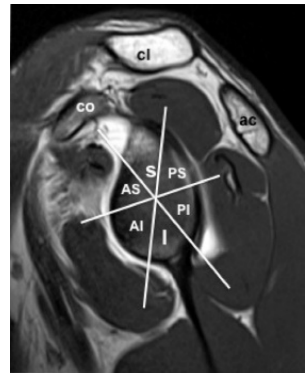

$1 \mathrm{~b}$

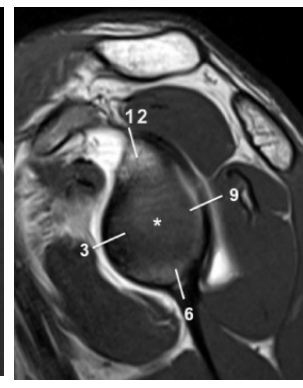

$1 \mathrm{c}$ that prevents anterior translation of the humeral head at 90 degrees of shoulder abduction. ${ }^{2}$ The glenoid fossa can be divided into six quadrants (Figure 1b) or labelled according to the numerals on a clock face (Figure 1c). The 12 o'clock position indicates the superior aspect of the glenoid fossa and the 6 o'clock position its inferior aspect. The anterior aspect of the glenoid is at the 1 to 5 o'clock positions, while the posterior aspect is the 7 to 11 o'clock positions, applicable for both left and right shoulders. The line between the 3 to 9 o'clock positions is known as the equator. On cross-sectional images, the glenoid labrum is typically triangular in shape (Figure 1d-e). However, its size, shape and configuration may be variable-being rounded, crescentic, cleaved or even absent. ${ }^{3}$

Figure 1: Sagittal MR arthrogram images of a normal left shoulder show 1a. the normal pearshaped configuration of the glenoid (asterisk) with a rim of labrum (arrows) at the margin of the glenoid. The glenoid rim exhibits hypointense signal. The glenoid can be labeled according to 1b. quadrants or 1c. the numerals on a clock face. 1d. Axial MR arthrogram image shows the normal triangular shape of the anterior labrum (white arrow). 1e. Coronal MR arthrogram image shows the normal superior labrum (white arrow) with the proximal long head of biceps tendon attached to it. [Key: $S=$ superior; $A S=$ anterosuperior; $A I=$ anteroinferior; $P S=$ posterosuperior; $P I=$ posteroinferior; $I=$ inferior; $c o=$ coracoid process; $c l=$ clavicle $; a c=$ acromion; subs = subscapularis muscle].

\section{IMAGING TECHNIQUES}

Radiography is the first-line imaging tool for evaluation of shoulder injury. It is easily available, cheap and fast to perform. The routinely performed views consist of anteroposterior (AP), Y-scapular (also known as "outlet") and axillary projections. In the emergency room, radiographs can easily be utilized to exclude fracture or joint dislocation. Post-reduction radiographs are useful to detect any bony Bankart lesion (Figure 2ab), Hill Sachs lesion and iatrogenic fracture. However, radiographs have poor sensitivity for the assessment of soft tissue structures. ${ }^{4}$ MR imaging serves as an excellent complementary modality to bridge this imaging gap. With its superior soft tissue resolution, the glenoid labrum is best studied using proton density fat-suppressed or fluid-sensitive fat-suppressed sequences in the oblique coronal and axial planes on conventional MR imaging without arthrography. ${ }^{5}$ Numerous studies have compared the overall diagnostic performance between the conventional MR imaging and direct MR arthrography, with the overall consensus being that the direct MR arthrography is superior for the diagnosis of labral tear. ${ }^{6}$ 


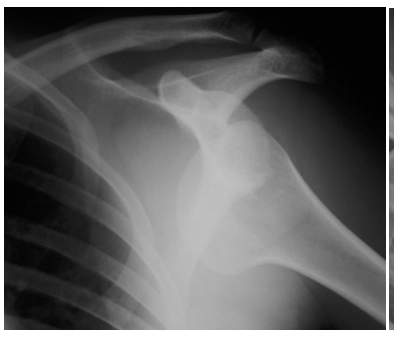

$2 \mathrm{a}$

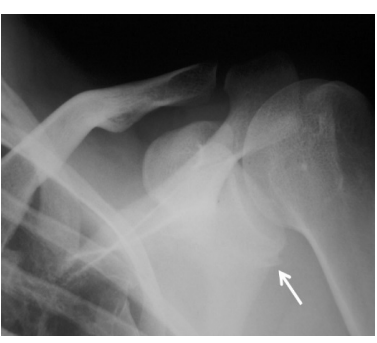

$2 \mathrm{~b}$
Figure $2 \boldsymbol{a} \boldsymbol{\&}$ 2b: A 23-year-old man with a bony Bankart lesion following a recent anterior glenohumeral dislocation. $2 \boldsymbol{a}$. Frontal left shoulder radiograph shows an anteroinferior dislocation of the humeral head. $2 \boldsymbol{b}$. Post-reduction radiograph shows a relocated left humeral head, but also reveals a bone fragment at the anteroinferior aspect of the glenoid fossa (white arrow) characteristic of a bony Bankart lesion.

Direct MR shoulder arthrography has a reported sensitivity and specificity of about $82-100 \%$ and $71-98 \%$, respectively, in diagnosing labral tear. ${ }^{5}$ Contrast agent that is directly injected into the shoulder joint enables optimal opacification of a torn labrum that may be otherwise be difficult to appreciate on a non-arthrographic MR imaging, particularly if the tear is undisplaced. This is especially true when the labral tear is chronic or normal anatomical variants (e.g. sublabral recess or foramen) are present and a labral tear needs to be excluded. ${ }^{5}$ However, MR arthrography is not necessary for patients with acute shoulder dislocation due to the glenohumeral joint effusion which provides natural intra-articular contrast.

Various approach techniques for shoulder arthrography have been previously described. We adopt a modified anterior approach targeting the triangular space at the superior humeral head that represents the rotator interval, which has a relative paucity of vital anatomical structures. The intention of this approach is to avoid the subscapularis tendon inferiorly, and intra-articular portion of the LHBT and supraspinatus tendon superiorly. Under fluoroscopic guidance, a small amount of iodinated contrast agent is used to confirm correct position of the needle before $10-15 \mathrm{ml}$ of gadolinium-based contrast agent in 1:200 dilution is administered into the shoulder joint. $^{7}$
Following shoulder arthrography, fatsuppressed T1-weighted images in three planes are acquired. The shoulder may be placed in an abduction and external rotation (ABER) position to create tension on the anteroinferior portion of the glenoid labrum and inferior IGHL. This technique allows better visualization of Bankart lesion, its variants and injury to the anterior IGHL. Other sequences such as non-fat-suppressed T1-weighted and fat-suppressed T2-weighted images are acquired to help in the overall assessment for other abnormalities affecting structures such as the bone and components of the rotator cuff. ${ }^{5}$

In cases where MR arthrography is contraindicated or failed, computed tomography (CT) arthrography may be a useful alternative; for example, in patients with metal hardware close to the joint, MRincompatible medical devices, and a history of claustrophobia. Disadvantages of CT arthrography include ionizing radiation and contrast-related adverse effects (e.g. allergic reaction to iodinated contrast agent). CT arthrography can be performed using either single (diluted iodinated contrast agent) or double-contrast technique (combination of diluted iodinated contrast agent and room air). The same technique of shoulder arthrography is employed to deliver the contrast material into the shoulder joint before image acquisition, with $150 \mathrm{mg} / \mathrm{ml}$ concentration of iodinated contrast agent recommended to achieve optimal contrast resolution. Small bony Bankart lesions are best detected on CT and can be used to supplement radiographs or MR imaging in selected cases. ${ }^{8}$ (Figure 3)

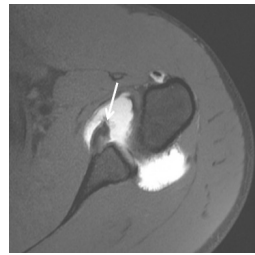

$3 \mathrm{a}$

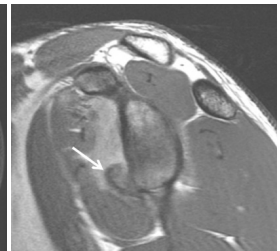

$3 \mathrm{~b}$

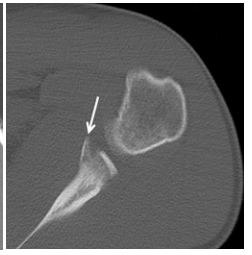

$3 \mathrm{c}$
Figure 3: Another case of a bony Bankart lesion depicted on MR arthrogram and CT of the left shoulder. 3a. Axial fat-suppressed and $\mathbf{3 b}$. sagittal T1-weighted MR arthrogram images show a torn anteroinferior labrum (white arrows) with an associated blunted glenoid rim. 3c. Corresponding axial CT image (taken in bone window) confirms a bony Bankart lesion (white arrow). 


\section{BANKART VARIANTS \\ LESIONS}

During anterior shoulder dislocation, the humeral head displaces anteroinferiorly, causingimpactioninjury ontotheanteroinferior glenoid labrum and posterolateral humeral head; the former leads to a Bankart lesion and the latter causes a Hill-Sachs lesion. The Bankart lesion is a tear of the anteroinferior labroligamentous complex (anteroinferior labrum together with the anterior band of the IGHL) that typically occurs between the 3 to 6 o'clock positions. The Bankart lesion can be classified into soft tissue Bankart (Figure 4) and bony Bankart lesions, with the latter including a fracture of the anteroinferior bony glenoid (Figure 3 ). In the bony Bankart lesion, a bony fragment of variable size is detached together with the anteroinferior labroligamentous complex. ${ }^{9}$ (Figure 5) Due to additional disruption of the scapular periosteum, the labrum is usually displaced from its normal anatomical position, such that it floats freely in the anterior joint space. ${ }^{9} \mathrm{~A}$ number of Bankart lesion variants have been described, including the Perthes lesion and anterior labroligamentous periosteal sleeve avulsion (ALPSA).

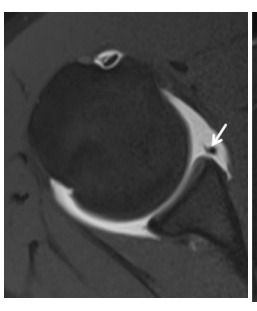

$4 \mathrm{a}$

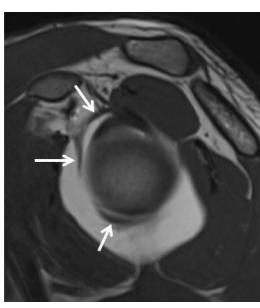

$4 b$

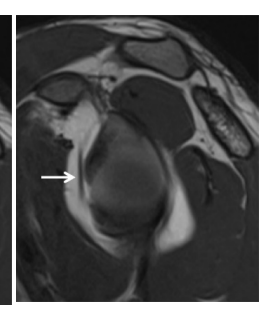

$4 c$
Figure 4: A 21-year-old man with a soft tissue Bankart lesion following two episodes of anterior glenohumeral dislocation. 4a. Axial fat-suppressed T1-weighted MR arthrogram image of the right shoulder shows a tear of the anteroinferior labrum (white arrow) without glenoid bone fracture. $\mathbf{4 b \boldsymbol { b }}$. Sagittal fat-suppressed T1-weighted MR arthrogram images show detachment of the antero-inferior glenoid labrum that extends superiorly (white arrows). Arthroscopy confirmed a soft tissue Bankart lesion located at the 2 o'clock to 6 o'clock positions.

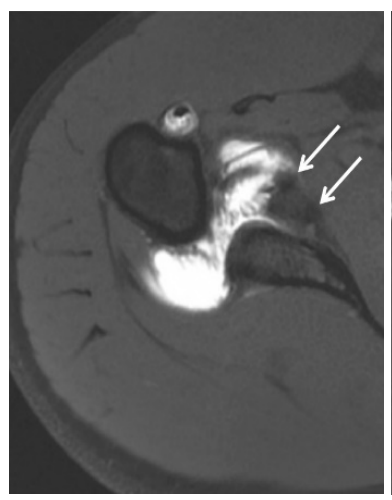

$5 \mathrm{a}$

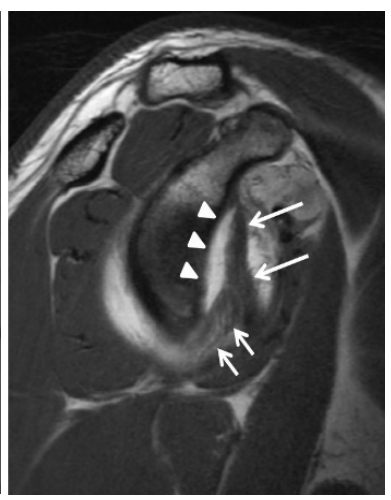

$5 \mathrm{~b}$
Figure 5: A 23-year-old man with a bony Bankart lesion following recent anterior glenohumeral dislocation. 5a. Axial fatsuppressed T1-weighted MR arthrogram image of the right shoulder shows a large detached triangular fragment consisting of a bony Bankart lesion and labroligamentous complex (white arrows). 5b. Sagittal T1weighted MR arthrogram image shows a large bony defect of the anterior glenoid (white arrowheads) located at the 2 o'clock to 6 o'clock positions. The bony (short white arrows) and labroligamentous (long white arrows) components of the detached fragment are also seen.

The Perthes lesion is a tear of the anteroinferior labroligamentous complex that remains connected an intact but medially-stripped scapular periosteum (Figure 6). The detached labrum may remain in a normal position, with the tear being masked by scar tissue and resynovialization, potentially making it difficult to be visualized on arthroscopy., ${ }^{9,10}$ The Perthes lesion is better seen with the patient's shoulder in an ABER position. In this position, the IGHL is under tension, enabling fluid or injected contrast agent to track beneath the stripped but intact scapular periosteum.

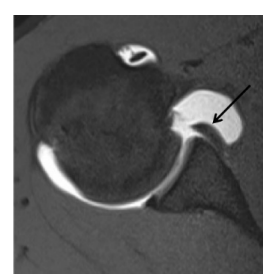

$6 \mathrm{a}$

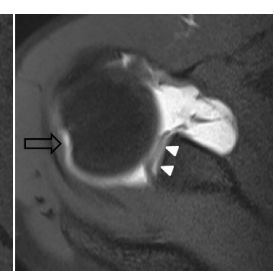

$6 \mathrm{~b}$

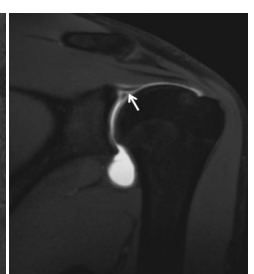

6c
Figure 6: 23-year-old man with Perthes lesion following multiple episodes of anterior glenohumeral dislocation. He also had 
an associated SLAP lesion. 6a. Axial fatsuppressed T1-weighted MR arthrogram image shows a tear of the anteroinferior labrum (black arrow) with a medially stripped but intact periosteum. 6b. A more superior MR image shows a small Hill-Sachs lesion (open arrow) as well as a tear of the superior labrum extending from anterior to posterior (arrowheads). 6c. Coronal fat-suppressed T1weighted MR arthrogram image shows the superior labral tear extending to the superior and lateral labrum (white arrow), giving rise to bucket-handle tear of the superior labrum. Arthroscopy revealed a Perthes lesion with bucket-handle tear of the superior labrum.

ALPSA (anteroinferior labroligamentous complex) can be considered a chronic variant of a Perthes lesion. ${ }^{10}$ The scapular periosteum is also stripped but intact, with its medial aspect still attached to the scapula. In ALPSA, however, there is medial displacement and inferior rotation of the avulsed labroligamentous complex, resulting in a mass-like lesion along the anterior surface of the scapular neck (Figure 7). The avulsed labrum may heal with scarring in a displaced position, with consequent chronic shoulder instability. ALPSA requires a different repair technique, compared to a classic Bankart lesion. ${ }^{11}$

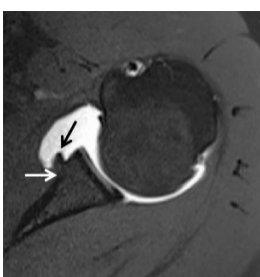

$7 \mathrm{a}$

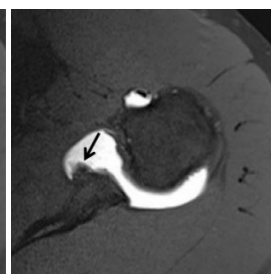

$7 \mathrm{~b}$

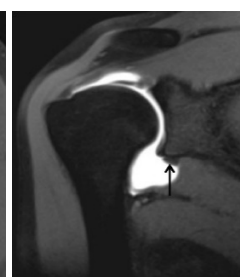

$7 \mathrm{c}$
Figure 7: ALPSA lesion in a 34-year-old man, a former rugby player, who presented with recurrent glenohumeral dislocation. $7 a$ \& 7b. Axial fat-suppressed T1-weighted MR arthrogram images of the left shoulder show detachment of the anteroinferior labrum with inferomedial displacement of the torn labrum (black arrow). The periosteum was medially stripped but still intact (white arrow). 7c. Coronal MR Image shows inferior displacement of the torn labrum (black arrow). A small Hill-Sachs lesion was present at the posterolateral humeral head (not shown).
Diagnosis of ALPSA lesion was confirmed operatively where superior extension of the detached labrum, involving the labrum from the 1 o'clock to 6 o'clock positions, was also found. The patient was discharged a day following arthroscopic repair; he underwent two weeks of post-operative rehabilitation and remained well on follow-up.

Depending on the severity of the labral tear and the number of recurrent dislocations, a Bankart lesion may sometimes extend superiorly to the superior labrum, with or without involvement of the LHBT (Figures 6b-c and 8), giving rise to a superior labral anterior posterior (SLAP) lesion. There are various classifications of SLAP lesions, with the combination of Bankart lesion and SLAP lesion being classified as SLAP V. Other associated injuries in anterior shoulder dislocation include avulsion of anterior IGHL, humeral avulsion of the glenohumeral ligament (HAGL) with or without bony avulsion, fracture, rotator cuff tear, vascular injury and neurovascular injury.

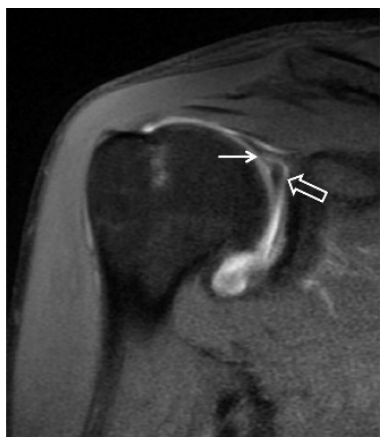

$8 \mathrm{a}$

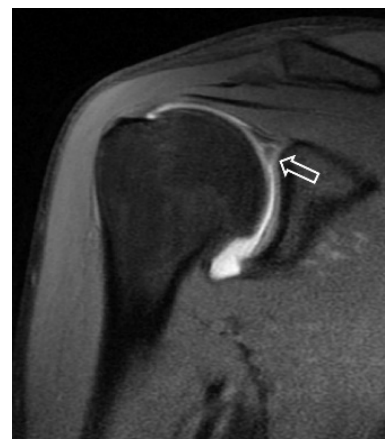

$8 \mathrm{~b}$
Figure 8: A 28-year-old man with a SLAP lesion associated with Bankart lesion (not shown) following recurrent episodes of anterior glenohumeral dislocations. $8 \boldsymbol{a} \boldsymbol{\&}$ 8b. Coronal fat-suppressed T1-weighted MR arthrogram images show superior extension of the labral tear to the proximal LHBT (white arrow) from the superior labrum (open arrows). These imaging findings were confirmed during arthroscopy.

\section{IMAGING PITFALLS}

In normal individuals, the base of the labrum is firmly attached to the bony glenoid rim, with some exceptions at the 11 to 3 o'clock 
positions. Normal labral variants may be identified at this region, such as sublabral recess (11 to 1 o'clock positions) (Figure 9), sublabral foramen or hole (1 to 3 o'clock positions) (Figure 10), Buford complex (1 to 3 o'clock positions) (Figure 11) and meniscoid labrum (superior labrum). In addition, a normal cleft may sometimes be seen between the labrum and the adjacent articular cartilage. Unlike a labral tear, the cleft does not extend to the entire width of the labrum. Meticulous assessment and knowledge of the typical appearances of these normal variants are required to avoid over- or misdiagnosis of labral tear. It is useful to remember that at the 3 to 6 o'clock positions, the presence of intraarticular contrast agent between the labrum and the underlying cartilage or bony glenoid almost always indicates a labral tear. ${ }^{12}$

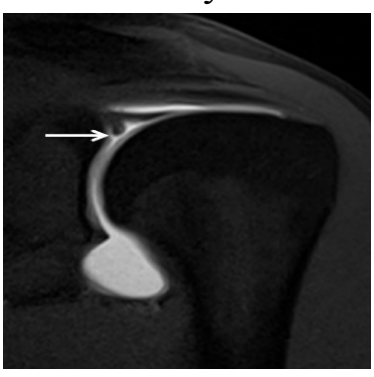

$9 \mathrm{a}$

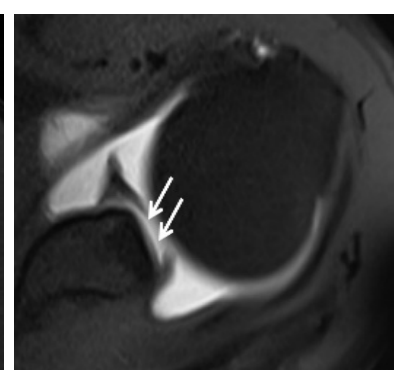

$9 \mathrm{~b}$
Figure 9: Normal anatomical variant of a sublabral recess. 9a. Coronal \& 9b. axial fat-suppressed T1-weighted MR arthrogram images show a smooth cleft at the base of the labrum that follows the contour of the glenoid cartilage from the 11 o'clock to 1 o'clock positions (white arrows), typical of a sublabral recess.

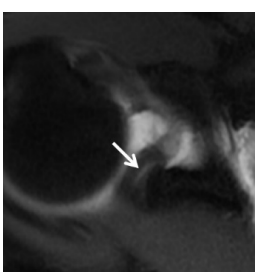

$10 \mathrm{a}$

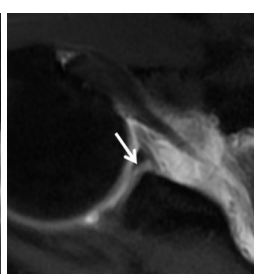

$10 \mathrm{~b}$

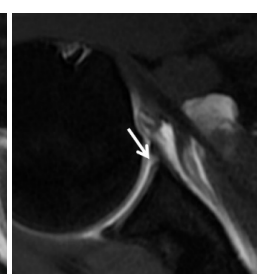

$10 \mathrm{c}$
Figure 10: Normal anatomical variant of a sublabral foramen. 10a, 10b \& 10c. Contiguous axial fat-suppressed T1-weighted $M R$ arthrogram images show a smooth cleft between the labrum and glenoid cartilage from the 1 o'clock to the 3 o'clock positions that courses medially (white arrows), consistent with a sublabral foramen.

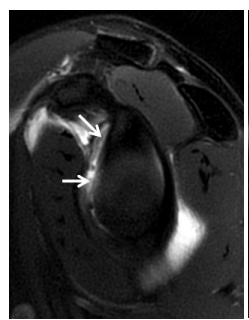

$11 \mathrm{a}$

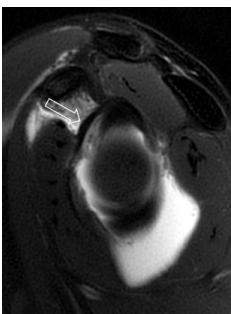

$11 b$

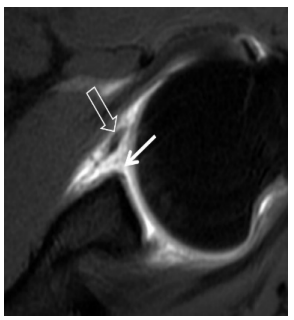

$11 \mathrm{c}$
Figure 11: Normal anatomical variant of a Buford complex. 11a \& 11b. Sagittal and 11c. axial fat-suppressed MR arthrogram images show absence of the anterosuperior labrum from the 1 o'clock to 3 o'clock positions (white arrows) associated with thickening of the medial glenohumeral ligament (open arrows). The combination of findings is characteristic for a Buford complex.

\section{SUMMARY}

The glenoid labrum is an important soft tissue structure that provides stability to the shoulder joint. When the labrum is injured, the shoulder is prone to recurrent dislocation and chronic instability.

\section{REFERENCES}

1. Howell SM, Galinat BJ. The glenoid-labral socket.Aconstrained articular surface. Clin Orthop Relat Res 1989;243(243):122-125. https://doi.org/10.1097/00003086198906000-00018.

2. Turkel SJ, Panio MW, Marshall JL, Girgis FG. Stabilizing mechanisms preventing anterior dislocation of the glenohumeral joint. $J$ Bone Joint Surg Am 1981;63(8):1208-1217. https://doi.org/10.2106/00004623198163080-00002

3. Al-Riyami AM, Lim BK, Peh WCG. Variants and pitfalls in MR imaging of shoulder injuries. Semin Musculoskelet Radiol 2014;18(1):36-44. https://doi.org/10.1007/978-3-31953496-1

4. Bianchi S, Prato N, Martinoli C, Derchi LE. Shoulder Radiography. In: Davies AM, 
Hodler J (editors). Imaging of the Shoulder: Technique and Applications. Berlin/ Heidelberg: Springer-Verlag 2006;3-13. https://doi.org/10.1007/3-540-30001-5

5. DeConinckT,NgaiSS, TafurM, ChungCB. Imaging the glenoidlabrum and labral tears. RadioGraphics 2016;36(6):1628-1647. https://doi.org/10.1148/rg.2016160020

6. Major NM, Browne J, Domzalski T, Cothran RL, Helms CA. Evaluation of the glenoid labrum with 3-T MRI: is intraarticular contrast necessary? Am $J$ Roentgenol 2011;196(5):1139-1144. https://doi.org/10.2214/AJR.08.1734

7. Chin TY, Campbell RSD. Arthrographic Technique Pitfalls. In: Peh WCG (editor). Pitfalls in Musculoskeletal Radiology. Springer International 2017:293-305. https://doi.org/10.1007/978-3-31953496-1

8. Fritz J, Fishman EK, Small KM et al. MDCT arthrography of the shoulder with datasets of isotropic resolution: Indications, technique, and applications. Am J Roentgenol 2012;198(3):635-646. https://doi.org/10.2214/AJR.11.7078
9. Woertler K. Shoulder Joint. In: Bohndorf K, Anderson MW, Davies AM, Imhof H, Wortler K. Imaging of Bones and Joints. A Concise, Multimodality Approach. Stuttgart: Thieme 2016:94-115.

10. Wischer TK, Bredella MA, Genant HK et al. Perthes lesion (a variant of the Bankart lesion): MRimaging and MR arthrographic findings with surgical correlation. Am $J$ Roentgenol 2002;178(1):233-237. https://doi.org/10.2214/ajr.178.1.1780233

11. Walz DM, Burge AJ, Steinbach L. Imaging of shoulder instability. Semin MusculoskeletRadiol2015;19(3):254-268. https://doi.org/10.1055/s-0035-1549319

12. Vossen JA, Palmer WE. Shoulder Injury: MRI pitfalls. In: Peh WCG (editor). Pitfalls in Musculoskeletal Radiology. Springer International 2017:293-305. https://doi.org/10.1007/978-3-31953496-1 